\title{
Standard Model Gauging of the WZW Term: Anomalies, Global Currents and pseudo-Chern-Simons Interactions
}

\author{
Jeffrey A. Harvey ${ }^{(a)}$, Christopher T. Hill ${ }^{(b)}$, Richard J. Hill ${ }^{(b)} *$ \\ ${ }^{(a)}$ Enrico Fermi Institute and Department of Physics \\ The University of Chicago, \\ Chicago, Illinois, 60637, USA \\ ${ }^{(b)}$ Fermi National Accelerator Laboratory \\ P.O. Box 500, Batavia, Illinois 60510, USA
}

(Dated: October 24, 2018)

\begin{abstract}
The standard model $S U(2)_{L} \times U(1)_{Y}$ gauging of the Wess-Zumino-Witten term requires a modified counterterm when background fields, needed to generate the full set of currents, are introduced. The modified counterterm plays an essential role in properly defining covariant global currents and their anomalies. For example, it is required in order to correctly derive the gauge invariant baryon number current and its anomalous divergence. The background fields can also be promoted to a description of the physical spin-1 vector and axial-vector mesons in QCD and the counterterm leads to novel interactions. These are (pseudo-) Chern-Simons terms, such as $\epsilon^{\mu \nu \rho \sigma} \omega_{\mu} Z_{\nu} \partial_{\rho} A_{\sigma}$ and $\epsilon^{\mu \nu \rho \sigma} \rho_{\mu}^{ \pm} W_{\nu}^{\mp} \partial_{\rho} A_{\sigma}$ that mediate new interactions between neutrinos and photons at finite baryon density.
\end{abstract}

PACS numbers: 11.15.-q, 12.15.-y, 12.38.Qk, 12.39.Fe, 13.15.+g, 13.40.-f, 14.70.Hp, 14.80.Mz, 95.85.Ry, 97.60.Jd

\section{INTRODUCTION}

The low-energy spectrum of QCD contains pseudoscalar mesons interpreted as the Nambu-Goldstone bosons (NGB's) of spontaneously broken chiral symmetry. $U\left(N_{f}\right)_{L} \times U\left(N_{f}\right)_{R}$ breaks to the diagonal, vector subgroup $U\left(N_{f}\right)_{V}$, with $N_{f}=2,3$ depending on whether just the $(u, d)$ quark symmetries, or the $(u, d, s)$ symmetries are included in the analysis. A complete low-energy chiral lagrangian describing the interactions of these meson states contains terms in the following three classes.

The first class consists of terms related to the familiar kinetic term:

$$
\mathcal{L}_{K}=\frac{f_{\pi}^{2}}{4} \operatorname{Tr}\left(D_{\mu} U^{\dagger} D^{\mu} U\right)+\ldots,
$$

where $f_{\pi} \approx 93 \mathrm{MeV}$, and $U=\exp \left[\left(2 i / f_{\pi}\right) \pi^{a} T^{a}\right]$ is a chiral matrix field transforming as $U \rightarrow e^{i \epsilon_{L}} U e^{-i \epsilon_{R}}$ under $U\left(N_{f}\right)_{L} \times U\left(N_{f}\right)_{R}$, with $e^{i \epsilon_{L, R}} \in U\left(N_{f}\right)_{L, R}$. The kinetic term can be made locally invariant under $U\left(N_{f}\right)_{L} \times U\left(N_{f}\right)_{R}$ transformations by including a complete set of gauge fields in the covariant derivative, $D U=\partial U-i A_{L} U+i U A_{R}$, with corresponding local gauge transformations for $A_{L, R}$. The ellipsis refers to an expansion in the number of derivatives, containing in the next order the Gasser-Leutwyler operators.

A second class of terms consists of those associated with symmetry breaking. This includes the operator $\operatorname{Tr}\left(M_{q} U\right)+$ h.c. where $M_{q}$ is the quark mass matrix, and

\footnotetext{
*Electronic address: harvey@theory.uchicago.edu, hill@fnal.gov,
} rjh@fnal.gov also the operator $\operatorname{det} U e^{i \theta}+$ h.c. which reflects the breaking of the axial $U(1)$ by instantons. We will largely ignore the effects of these first two classes of operators in what follows.

A third class of operators comprise the Wess-ZuminoWitten $(\mathrm{WZW})$ term, $\Gamma_{W Z W}(U)$ 1, 2]. This is a topological object and it arises "holographically" when the $D=4$ manifold of spacetime is viewed as the boundary of a $D=5$ manifold [2]. The WZW term is intimately connected to the anomaly structure of QCD. When coupled to classical background gauge fields $A_{L}, A_{R} \in U\left(N_{f}\right)_{L, R}$ the variation of $\Gamma_{W Z W}\left(U, A_{L}, A_{R}\right)$ under local $U\left(N_{f}\right)_{L} \times$ $U\left(N_{f}\right)_{R}$ is non-zero, and reproduces the anomalies of the underlying theory of quarks $[1,2,2,3,4,45,6,6,[8,9]$.

Moreover, $\Gamma_{W Z W}$ lifts a spurious parity symmetry in the chiral lagrangian, locking the pion parity to that of space (it performs a similar task in Little Higgs theories by breaking spurious $T$-parity [10]). It mediates processes such as $K \bar{K} \rightarrow 3 \pi$ which are allowed by QCD but would be forbidden in the low-energy chiral lagrangian theory if we kept only the first two classes of terms and ignored the WZW term. The WZW term can also be coupled to physical gauge fields, like the photon. This leads to a correct description of the process $\pi^{0} \rightarrow 2 \gamma$, that is otherwise forbidden by the extension of the spurious parity to gauge fields. Thus the WZW term generates an essential part of the physics, and should be placed on the same footing as the other terms in the chiral lagrangian.

In this paper we are interested in the gauged WZW term with physical gauge fields coupled to the flavor symmetries of the quarks. In general, to achieve an anomaly free gauge theory we can either gauge an anomaly free subgroup of $U\left(N_{f}\right)_{L} \times U\left(N_{f}\right)_{R}$, or cancel anomalies between the chiral lagrangian and a "lepton sector". The 
former case arises when gauging only electromagnetism in the QCD chiral Lagrangian, and is perhaps the most familiar application of a gauged WZW term. However, it is the latter situation that arises for the $S U(2)_{L} \times U(1)_{Y}$ electroweak gauge group of the standard model. This leads to additional issues that need to be addressed, due to the fact that $S U(2)_{L} \times U(1)_{Y}$ resides in a nondiagonal subgroup of the chiral symmetry group. We are forced to revisit the counterterm structure of the Wess-ZuminoWitten term. Ultimately we are led to a new counterterm, and in turn, to new physics. This is the focus of the present paper.

In addition to the fundamental gauge fields of the standard model, i.e., the $W, Z$ and $\gamma$, the theory must be consistent if we include background fields that couple to the currents of the chiral lagrangian. These background fields are theoretically essential because they allow us to determine the correct form of the global chiral currents and their anomalies.

In what follows we will denote generic fundamental gauge fields by $A$ and background vector and axial-vector fields by $B$. The $B$ fields will be assumed to transform covariantly under the fundamental local gauge group of the standard model. Varying the effective action with respect to $B^{a}$ generates the associated current $J^{a}$. Varying the background fields locally, $\delta B=d \epsilon+\ldots$, as if they were fundamental gauge fields, generates the anomalous divergence of the associated global current via the WZW term. For example, a background field can be introduced with the quantum numbers of the $\omega$ meson, coupling to the quark baryon current. This automatically implies its coupling to the Goldstone-Wilczek skyrmionic baryon number current 2, 11] via the WZW term.

Introducing the $B$ fields leads, however, to the following subtle issue. When we have a set of fundamental gauge fields $A$, such as the standard model $W, Z$ and $\gamma$, and we then turn on the background $(B)$ fields, we find that new anomalies appear in the gauged $(A)$ currents that were previously absent. We can maintain the fundamental $(A)$ gauge invariance, however, if we can find a local counterterm, a functional of $A$ and $B$, which cancels these new anomalies.

The logic of this situation is identical to that of QED, underlying the original covariant anomaly first computed by Adler [5]. If we compute triangle diagrams for QED with a vector photon $A_{\mu}$ coupled to a massless electron as $A^{\mu} \bar{\psi} \gamma_{\mu} \psi$ then we do obtain a conserved vector current, and the divergence of the axial current is the consistent axial current anomaly [28]:

$$
\partial^{\mu} j_{\mu}^{5}=\frac{1}{48 \pi^{2}} \epsilon_{\mu \nu \rho \sigma} F^{\mu \nu} F^{\rho \sigma} .
$$

However, if we introduce a classical background field $B_{\mu}$ with the coupling $B^{\mu} j_{\mu}^{5}$, then we find that the vector current is no longer conserved, but develops a mixed anomaly $\propto \epsilon_{\mu \nu \rho \sigma} F_{A}^{\mu \nu} F_{B}^{\rho \sigma}$. It is essential that gauge invariance, i.e., vector current conservation, be maintained for any background field $B$, and we thus re- quire a counterterm. The counterterm takes the form $\left(1 / 6 \pi^{2}\right) \epsilon_{\mu \nu \rho \sigma} B^{\mu} A^{\nu} \partial^{\rho} A^{\sigma}$. When added to the action, it modifies the definitions of the currents. For example, the gauged vector current is $\delta S / \delta A_{\mu}$, and the global axial current is $\delta S / \delta B_{\mu}$, where $S$ is the action. The currents acquire corrections from the counterterm. This leads to a conserved modified vector current for any background field $B$ and the familiar covariant anomaly for the modified axial current [29],

$$
\partial^{\mu} j_{\mu}^{5}=\frac{1}{16 \pi^{2}} \epsilon_{\mu \nu \rho \sigma} F^{\mu \nu} F^{\rho \sigma} .
$$

The problem of maintaining gauge invariance of the WZW term for arbitrary background fields when the fundamental gauge fields are vectorlike, i.e., $A_{L}=A_{R}=A$, has a well-known solution: the "Bardeen counterterm," given by: $-\Gamma_{W Z W}\left(1, A+B_{L}, A+B_{R}\right)$. This is essentially a generalization of the aforementioned counterterm of QED. Adding this counterterm to the WZW term, $\Gamma_{W Z W}\left(U, A+B_{L}, A+B_{R}\right)$, ensures that vector currents are conserved for any $B_{L, R}$ background fields. We note that this procedure kills off an entire class of non-pionic interactions in the bare WZW term, such as $\epsilon_{\mu \nu \rho \sigma} A^{\mu} B_{L, R}^{\nu} \partial^{\rho} A^{\sigma}+\ldots$, which we call "pseudo-ChernSimons" (pCS) terms [30]. The special case of vectorlike gauging may thus lead to the intuition that pCS terms are somehow unphysical and don't appear in the full theory. However, this would be a false impression.

When gauging nondiagonal (non-vectorlike) subgroups such as $S U(2)_{L} \times U(1)_{Y}$, the Bardeen counterterm does not render the theory gauge $(A)$ anomaly free. We will show, however, that there always exists a new local counterterm that does maintain gauge invariance for the gauging of any subgroup in the general background of spin-1 classical $(B)$ fields. We give the explicit solution for the new counterterm in the general case and apply it in specific cases.

Once the new counterterm is incorporated into the WZW term two important things happen. First, the global currents, as generated by local variations of the appropriate background fields, become proper covariant objects. The anomalies of these currents are the covariant anomalies of the theory. The global baryon current and its anomaly provides an important example of this phenomenon. The current is modified from the GoldstoneWilczek form, in the presence of gauge fields, and becomes a gauge invariant operator. Its anomaly, arising from a local gauge transformation of the WZW term in the background $\omega$ field, $\delta \omega_{\mu}=\partial_{\mu} \epsilon$, yields the correct covariant baryon current anomaly. We note that there are also corrections involving the background fields themselves, e.g., including a term $\epsilon^{\mu \nu \rho \sigma} F_{W \mu \nu}^{a} D_{\rho} \rho_{\sigma}^{a}$ where $\rho_{\mu}^{a}$ is the background field with quantum numbers of the $\rho$ meson and $F_{W \mu \nu}^{a}$ is the $S U(2)_{L}$ field strength.

Second, there are now uncancelled pCS term interactions involving the fundamental gauge fields and the spin1 background fields that do not involve the pions. These pCS terms contain observable new physics. 
Indeed, the classical background $(B)$ fields can be promoted to describe the physical vector meson fields of QCD, i.e., the $\rho, \omega, a_{1}, f_{1}$ and so on. It is important to realize the distinction between classical background fields and physical spin-1 mesons: the former would describe "pointlike particles," present on all scales of the theory, while the physical spin-1 mesons have form-factors and decouple from the high energy quark loops. They are only part of an effective low energy theory. However, at low energies the physical spin-1 mesons can be viewed as coupling to the global currents and they thus behave like the $B$ fields. Anomalous physical processes involving them can be described by the WZW term. The pCS terms are a new part of the physics in the WZW term, involving exclusively the spin-1 mesons and gauge fields.

There are many formal issues that must be faced in the description of the vector mesons as propagating physical particles, and treating them as gauge fields [7, 12] incurs subtleties. However, phenomenologically successful treatments of processes that involve the spin-1 mesons and probe the anomalies encoded into the WZW term, such as $\omega, \rho \rightarrow \pi \gamma, \omega \rightarrow 3 \pi$, etc.., do flourish in the literature. We will postpone the detailed discussion of these issues to a subsequent paper [13].

Notably, from the new counterterm we obtain an interaction of the form $\epsilon^{\mu \nu \rho \sigma} \omega_{\mu} Z_{\nu} F_{\rho \sigma}$ where $\omega$ is the omega meson background field, $Z$ the $Z$-boson in unitary gauge, and $F_{\mu \nu}$ the photon field strength [14]. This interaction survives as an essential consequence of the non-diagonal standard model gauge structure and the new counterterm.

The outline of this paper is as follows. In Section II we construct a schematic version of the standard model, i.e., a "toy" model, in which the WZW term is nontrivial, but much simpler than in the standard model. This model consists of a single color and flavor of quark, and a single lepton. We gauge the $U(1)_{L} \times U(1)_{R}$ quark and lepton flavor symmetries by introducing a " $Z$ " associated with $U(1)_{L}$ and a photon " $A$ " associated with $U(1)_{V}$. The gauge anomalies cancel between the quark and the lepton sectors, as in the standard model. We then integrate out the quark with a large chirally invariant "constituent" mass term, $m_{q} \bar{q}_{L} q_{R} e^{i \phi / f}$, containing a "pion" $\phi$. This generates the WZW term involving $\phi, Z$ and $A$, which is easy to derive.

We then introduce the " $\omega$ " vector meson as a background field coupled to the baryon current. We show that new anomalies arise in the gauged currents and then construct the counterterm that cancels these anomalies. We discover that pCS terms such as $\epsilon_{\mu \nu \rho \sigma} \omega^{\mu} Z^{\nu} F^{\rho \sigma}$ remain in the physical WZW term [14]. Variation of the $\omega$ field generates the global baryon current and associated covariant anomaly.

In Section III we consider the general problem of a chiral lagrangian for a theory in which the chiral flavor symmetry $G$ is spontaneously broken to a subgroup $H$ while at the same time we gauge a subgroup $G^{\prime} \subset G$. We show how to construct the counterterm that maintains the $G^{\prime}$ gauge anomaly structure in the presence of background spin-1 fields. For a diagonal gauge group $G^{\prime} \subset H$, this reduces to the Bardeen counterterm (modulo gauge invariant operators).

In Section IV] we show that this counterterm plays a crucial role in the derivation of global symmetry currents and their anomalies. The anomalous baryon current provides an important application of this formalism. At the chiral lagrangian level we obtain a gauge invariant baryon current from the WZW term with the new counterterm. We examine the global symmetries that are neutral under the gauged symmetries (i.e., for which there is no explicit symmetry breaking by gauging), and find the general form of the global anomalies for arbitrary background fields.

In Section $\nabla$ we apply these ideas to derive the WZW term (including counterterms) for the $S U(2)_{L} \times U(1)_{Y}$ gauging of the $U(2)_{L} \times U(2)_{R}$ chiral symmetry of QCD in a background of the spin- 1 vector mesons $\rho, \omega, a_{1}$, and $f_{1}$. Physical applications of these ideas are mentioned, but the details are postponed to a subsequent paper [13]. For example, anomaly mediated neutrino photon interactions arise from the $\epsilon_{\mu \nu \rho \sigma} \omega^{\mu} Z^{\nu} F^{\rho \sigma}$ pCS interaction, and provide a possible explanation for excess events seen in the MiniBooNE experiment [13, 14, 15]. In SectionVI we conclude and outline some further implications of these ideas.

The $S U(2)_{L} \times U(1)_{Y}$ gauging of the QCD WZW term in general backgrounds has not, to our knowledge, been previously developed. Pseudo-Chern Simons terms with arbitrary coefficients have previously been appended to the effective Lagrangian in an ad hoc manner [16, 17, 18], with various phenomenological constraints on the coefficients. The advantage of our approach is that we predict the coefficients of such interactions in terms of the strong coupling constants of the QCD vector and axial-vector mesons. Our observations about pCS terms and global anomalies apply to general chiral lagrangian models, and are new.

\section{SCHEMATIC STANDARD MODEL WITH PCS INTERACTIONS}

\section{A. The WZW term}

We now construct a schematic model that exhibits in a simple way the necessity of adding new counterterms to the WZW term, and the existence of pCS terms. This model involves only abelian gauge groups, but is constructed in close analogy to QCD and the $S U(2)_{L} \times$ $U(1)_{Y}$ electroweak sector of the standard model. It will form the basis for the general discussion of the $S U(2)_{L} \times U(1)_{Y}$ gauging of the $U(2)_{L} \times U(2)_{R}$ chiral lagrangian of pions.

We consider a theory with a single $\left(N_{c}=1\right)$ "quark" $q$ and a single "lepton" $\ell$. We introduce $U(1)_{L}$ and $U(1)_{R}$ fundamental gauge fields $A_{L}$ and $A_{R}$ into the quark ac- 
tion:

$$
S_{q}=\int d^{4} x \bar{q}_{L}\left(i \not \partial+\not A_{L}\right) q_{L}+\bar{q}_{R}\left(i \not \partial+\not A_{R}\right) q_{R} .
$$

$S_{q}$ has gauge currents,

$$
\begin{aligned}
& J_{\mu}^{(B)_{L}}=\frac{\delta S_{q}}{\delta A_{L}^{\mu}}=\bar{q}_{L} \gamma_{\mu} q_{L}, \\
& J_{\mu}^{(B)_{R}}=\frac{\delta S_{q}}{\delta A_{R}^{\mu}}=\bar{q}_{R} \gamma_{\mu} q_{R},
\end{aligned}
$$

that are anomalous:

$$
\begin{aligned}
& \partial^{\mu} J_{\mu}^{(B)_{L}}=-\frac{1}{24 \pi^{2}} \epsilon_{\mu \nu \rho \sigma} \partial^{\mu} A_{L}^{\nu} \partial^{\rho} A_{L}^{\sigma}, \\
& \partial^{\mu} J_{\mu}^{(B)_{R}}=\frac{1}{24 \pi^{2}} \epsilon_{\mu \nu \rho \sigma} \partial^{\mu} A_{R}^{\nu} \partial^{\rho} A_{R}^{\sigma} .
\end{aligned}
$$

Here we use the consistent anomalies that arise from Weyl spinor triangle diagrams.

To cancel these anomalies we gauge the "lepton" sector:

$$
S_{\ell}=\int d^{4} x \bar{\ell}_{L}\left(i \not \partial-\not A_{L}\right) \ell_{L}+\bar{\ell}_{R}\left(i \not \partial-A_{R}\right) \ell_{R} .
$$

Note that the relative signs of the quark and lepton couplings imply that $A_{L}$ couples to $B-L$ for the left-handed fields, which we denote by $(B-L)_{L}$, while $A_{R}$ couples to $(B-L)_{R}$. Taken together the gauge anomalies cancel between the quark and lepton sectors in the $B-L$ currents:

$$
\begin{aligned}
& \partial_{\mu}\left(\bar{q} \gamma^{\mu} q_{L}-\bar{\ell} \gamma^{\mu} \ell_{L}\right)=\partial^{\mu} J_{\mu}^{(B-L)_{L}}=0, \\
& \partial_{\mu}\left(\bar{q} \gamma^{\mu} q_{R}-\bar{\ell} \gamma^{\mu} \ell_{R}\right)=\partial^{\mu} J_{\mu}^{(B-L)_{R}}=0 .
\end{aligned}
$$

Anomalies remain in the ungauged $B+L$ currents, imitating the structure of the standard model.

We are interested in an analogy to hadronic physics and the chiral lagrangian of QCD. Thus, we want to spontaneously break the $U(1)_{L} \times U(1)_{R}$ of the quark sector to $U(1)_{V}$. We can do so by introducing a constituent quark mass term containing an NGB denoted by $\phi$ :

$$
m_{q} e^{i \phi / f} \bar{q}_{L} q_{R}+\text { h.c. }
$$

Here $\phi / f$ is the analog of $\pi / f_{\pi}$ in QCD.

Technically, in this model the NGB, $\phi$, would be eaten by the linear combination $Z=A_{L}-A_{R}$, which then becomes massive. This is the analog of symmetry breaking in technicolor theories. Alternatively, we can imagine an additional Higgs scalar field that gives the $Z$ its mass by developing a VEV $v$. $Z$ then acquires a longitudinal component, $\chi$, from the phase of the Higgs, $Z \rightarrow Z-\partial \chi / v$. In this case a dynamical NGB remains in the low-energy spectrum which is a linear combination of $\chi$ and $\phi$ (mainly the $\phi$ field in the $v \gg f$ limit). We will assume the Higgs mechanism is present, allowing the $Z$ to acquire mass, but we need not explicitly write the $\chi$ lagrangian.
In what follows we will use the abbreviated notation of differential forms, so that for example $\int d^{4} x \epsilon_{\mu \nu \rho \sigma} A^{\mu} B^{\nu} \partial^{\rho} C^{\sigma}=\int A B d C$.

Under $U(1)_{L} \times U(1)_{R}$ gauge transformations we have:

$$
\begin{aligned}
& q_{L} \rightarrow e^{i \epsilon_{L}} q_{L}, \quad \ell_{L} \rightarrow e^{-i \epsilon_{L}} \ell_{L}, \quad \delta A_{L}=d \epsilon_{L}, \\
& q_{R} \rightarrow e^{i \epsilon_{R}} q_{R}, \quad \ell_{R} \rightarrow e^{-i \epsilon_{R}} \ell_{R}, \quad \delta A_{R}=d \epsilon_{R}, \\
& \delta \phi=f\left(\epsilon_{L}-\epsilon_{R}\right) \text {. }
\end{aligned}
$$

The gauge transformations acting purely on the quark sector are anomalous and induce a shift in the quark effective action, $\delta S=\int\left(\partial_{\mu} \epsilon\right) J^{\mu}=-\int \epsilon \partial_{\mu} J^{\mu}$ :

$$
\delta S_{q}=\frac{1}{24 \pi^{2}} \int \epsilon_{L} d A_{L} d A_{L}-\epsilon_{R} d A_{R} d A_{R} .
$$

This is, of course, cancelled by the anomalous shift in the lepton effective action:

$$
\delta S_{\ell}=-\frac{1}{24 \pi^{2}} \int \epsilon_{L} d A_{L} d A_{L}-\epsilon_{R} d A_{R} d A_{R} .
$$

Hence, overall we have a non-anomalous gauge symmetry and conserved gauged currents as stated in Eq.(8).

We now consider a large $m_{q}$ limit and integrate out the quarks (which imitates the effect of confinement; related examples have been discussed in [19, 20]). We are left with an effective action:

$$
\Gamma_{W Z W}\left(U, A_{L}, A_{R}\right)+\ldots,
$$

where $\Gamma_{W Z W}$ is the Wess-Zumino-Witten term and the ellipsis refers to non-topological terms, such as renormalized $\phi$ kinetic terms. $\Gamma_{W Z W}$ is a functional of $U=e^{i \phi / f}$ and the gauge fields, $A_{L}$ and $A_{R} . \Gamma_{W Z W}$ generates the same anomalies as the quark action in Eq.(11) under the gauge transformations of Eq.(10).

It is easy to construct the WZW term, by arranging a set of operators that generate the independent $L$ and $R$ consistent anomalies. We readily obtain:

$$
\begin{aligned}
\Gamma_{W Z W} & =\frac{1}{24 \pi^{2}} \int\left[A_{L} A_{R} d A_{L}+A_{L} A_{R} d A_{R}\right. \\
& \left.+\frac{\phi}{f}\left(d A_{L} d A_{L}+d A_{R} d A_{R}+d A_{L} d A_{R}\right)\right] .
\end{aligned}
$$

It can easily be checked that, under the gauge transformations (10), we have:

$$
\delta \Gamma_{W Z W}=\delta S_{q},
$$

with $\delta S_{q}$ from Eq.(11).

Note that Eq.(14) can be obtained from the expression for the $U(N) \times U(N) / U(N)$ WZW term discussed in Ref. [7], by taking $N=1$. It can also be straightforwardly derived "holographically" from the Chern-Simons term and Dirac determinant of a compactified $D=5$ $U(1)$ gauge theory in which $\phi \sim A_{5}$, as in Ref. 21]. 


\section{B. Introduction of the $\omega$}

We now introduce a classical background field coupled to the "baryon number" in the quark sector. We denote this field by $\omega$ in analogy to the $\omega$ meson of QCD which couples to the baryon current. The WZW action becomes:

$$
\Gamma_{W Z W}\left(U, A_{L}+\omega, A_{R}+\omega\right)
$$

Note that $\omega$ is invariant under $U(1)_{L} \times U(1)_{R}$ gauge transformations. In analogy to QCD we view $\omega$ as part of the strong interactions, and do not couple it to the lepton sector. With $\omega$ appearing only in the quark sector, we then find that the theory now contains anomalies under local $U(1)_{L} \times U(1)_{R}$ gauge transformations.

From Eq.11] we see that:

$$
\begin{array}{r}
\delta\left(\Gamma_{W Z W}+S_{\ell}\right)=\frac{1}{24 \pi^{2}} \int \epsilon_{L}\left[2 d A_{L} d \omega+(d \omega)^{2}\right] \\
-\epsilon_{R}\left[2 d A_{R} d \omega+(d \omega)^{2}\right] .
\end{array}
$$

The gauge symmetry, and the internal consistency of the theory, is apparently spoiled by the inclusion of $\omega$. If, however, we can find a local counterterm, a functional of $A_{L} A_{R}$ and $\omega$, to add to the lagrangian that restores $U(1)_{L} \times U(1)_{R}$ gauge invariance in the presence of a background $\omega$, then the theory can be made consistent, as in the case of QED, summarized in the Introduction.

The desired counterterm is readily constructed:

$$
\begin{array}{r}
\Gamma_{c}=-\frac{1}{24 \pi^{2}} \int\left[-2 \omega A_{R} d A_{R}-\omega A_{R} d \omega\right. \\
\left.+2 \omega A_{L} d A_{L}+\omega A_{L} d \omega\right] .
\end{array}
$$

This counterterm is a necessary part of the low energy theory when $\omega$ is introduced into $\Gamma_{W Z W}$. Adding $\Gamma_{c}$ to $\Gamma_{W Z W}$ we see that the new $\omega$ dependent terms in Eq.(17) are now cancelled under a gauge transformation. If $\omega$ is an arbitrary classical background field that couples also to quarks at high energies, then this counterterm is required in the high energy action, $S_{q}$, as well.

The full WZW term is now given by the sum of $\Gamma_{W Z W}$ and the counterterm:

$$
\begin{aligned}
\Gamma_{W Z W}^{\text {full }} & =\Gamma_{W Z W}\left(\phi, A_{L}+\omega, A_{R}+\omega\right)+\Gamma_{c}\left(A_{L}, A_{R}, \omega\right) \\
& \equiv \Gamma_{W Z W}\left(\phi, A_{L}, A_{R}\right)+\Gamma_{\omega}\left(\phi, A_{L}, A_{R}, \omega\right), \quad(19)
\end{aligned}
$$

where we've isolated the interactions involving $\omega$ into $\Gamma_{\omega}$ :

$$
\begin{aligned}
\Gamma_{\omega}= & \frac{1}{8 \pi^{2}} \int\left[\frac{\phi}{f}\left(d A_{L} d \omega+d A_{R} d \omega+d \omega d \omega\right)\right. \\
& -\omega A_{L} d A_{L}+\omega A_{R} d A_{R}+\omega A_{R} d A_{L} \\
& \left.-\omega A_{L} d A_{R}+\omega A_{R} d \omega-\omega A_{L} d \omega\right] .
\end{aligned}
$$

We thus see that pCS terms, such as $\omega A_{L} d A_{L}$, now appear in the complete effective action. $\Gamma_{W Z W}\left(\phi, A_{L}, A_{R}\right)$ generates the original anomalies $\propto-d A_{L} d A_{L}+d A_{R} d A_{R}$ that are cancelled by the leptons. $\Gamma_{\omega}$ governs interactions of $\phi, A_{L}$ and $A_{R}$ with $\omega$ (these are analogous to anomalous interactions in QCD such as $\omega \rightarrow \pi^{0} \gamma$ ).

Since $\Gamma_{\omega}$ generates no new gauge anomalies, it must be itself a gauge invariant operator. Moreover, under local shifts in $\omega$, i.e., $\delta \omega=d \epsilon$ this term generates the global baryon current anomaly:

$$
\begin{aligned}
& \delta \Gamma_{W Z W}^{\text {full }}=\delta \Gamma_{\omega}= \\
& \frac{1}{8 \pi^{2}} \int \epsilon\left[d A_{L} d A_{L}-d A_{R} d A_{R}+d A_{L} d \omega-d A_{R} d \omega\right]
\end{aligned}
$$

or:

$$
\begin{gathered}
\partial_{\mu} J^{\mu}=-\frac{1}{8 \pi^{2}} \epsilon_{\mu \nu \rho \sigma}\left[\partial^{\mu} A_{L}^{\nu} \partial^{\rho} A_{L}^{\sigma}-\partial^{\mu} A_{R}^{\nu} \partial^{\rho} A_{R}^{\sigma}\right. \\
\left.+\partial^{\mu} A_{L}^{\nu} \partial^{\rho} \omega^{\sigma}-\partial^{\mu} A_{R}^{\nu} \partial^{\rho} \omega^{\sigma}\right] .
\end{gathered}
$$

Here $J^{\mu}$ is the baryon number current, given by:

$$
\begin{aligned}
& J_{\mu}=\frac{\delta}{\delta \omega^{\mu}} \Gamma_{W Z W}^{\mathrm{full}}= \\
& \begin{array}{r}
-\frac{1}{8 \pi^{2}} \epsilon_{\mu \nu \rho \sigma}[ \\
{\left[A_{L}^{\nu} \partial^{\rho} A_{L}^{\sigma}-A_{R}^{\nu} \partial^{\rho} A_{R}^{\sigma}+A_{L}^{\nu} \partial^{\rho} A_{R}^{\sigma}\right.} \\
-A_{R}^{\nu} \partial^{\rho} A_{L}^{\sigma}+A_{L}^{\nu} \partial^{\rho} \omega^{\sigma}-A_{R}^{\nu} \partial^{\rho} \omega^{\sigma} \\
-\partial^{\nu}(\phi / f)\left(\partial^{\rho} A_{L}^{\sigma}+\partial^{\rho} A_{R}^{\sigma}+2 \partial^{\rho} \omega^{\sigma}\right) \\
\left.\quad+\partial^{\nu}\left(A_{R}^{\rho} \omega^{\sigma}-A_{L}^{\rho} \omega^{\sigma}\right)\right] .
\end{array}
\end{aligned}
$$

where the last two lines contribute zero to the anomaly.

We thus see that the anomalous divergences of currents associated with global symmetries are now defined in the presence of arbitrary background fields through the variation of a consistent, gauge-invariant action. Note that the baryon number anomaly is modified in the presence of the background $\omega$ field by the $d A d \omega$ terms. Normally, we think of the global charges and their anomalies as defined in the limit $\omega \rightarrow 0$, but we are free to consider the background field corrections once the gauged currents are defined to be conserved. In summary: The WZW term requires the prescribed counterterm to recover the correct form of the baryon current anomaly. The $p C S$ terms are a consequence of this structure and generate new physical interactions.

From Eq.(20) we can anticipate an interesting new physical application of anomaly physics in the real world as described in Ref. [14]. We let $A_{L}=Z+A$ and $A_{R}=A$, where $Z$ is the analog of the $Z$-boson and $A$ the photon. Then we obtain from Eq.(20) the following pCS interaction term:

$$
\begin{aligned}
& \Gamma_{W Z W}^{\mathrm{full}}=\Gamma_{W Z W}\left(\phi, A_{L}, A_{R}\right) \\
& \quad-\frac{1}{8 \pi^{2}} \int[\omega(2 d A+d Z)+\omega d \omega]\left(Z-\frac{d \phi}{f}\right) .
\end{aligned}
$$

Gauge invariance of the photon is manifest, as it must be, since $2 d A \equiv F$ is the electromagnetic field strength. 
The $Z$ boson is associated with spontaneous symmetry breaking. We see that the gauge shift in $Z, \delta Z=d \epsilon$, is compensated by $\delta \phi=f \epsilon$ which confirms gauge invariance (again, if a Higgs mechanism is not present to give $Z$ its mass, then it will eat the $\phi$ field as in technicolor theories).

The interaction in Eq.(24) is a term in the low-energy effective theory describing physics at energy scales below the quark mass. It contains the massive gauge field $Z$, and if $M_{Z} \gg m_{q}$, we integrate out the $Z$ to derive a set of couplings involving only light fields as in Ref. 14]. The $\omega Z d A$ term leads to a novel neutrino-photon interaction in nucleons or at finite baryon density, which may be relevant to various experiments and astrophysical processes [14].

\section{THE COUNTERTERM FOR GENERAL GAUGING}

The schematic model illustrates a problem that can be posed more generally as follows. Consider a "quark sector" with a global (chiral) flavor symmetry $G$ and a subgroup $G^{\prime} \subset G$ which is gauged. In general, $G^{\prime}$ contains anomalies coming from the quark sector, so we further assume a lepton sector coupled to the gauge fields of $G^{\prime}$, which cancels the quark sector anomalies. The quarks are confined, or decoupled, and the flavor symmetry $G$ is broken spontaneously to a subgroup $H$, giving rise to NGB's that are elements of the coset space $G / H$. Some of the NGB's may be eaten by gauge fields, or the gauge fields may acquire mass from a Higgs sector.

As a concrete realization of this we can consider the $(u, d)$ quarks with flavor symmetry $G=S U(2)_{L} \times$ $S U(2)_{R} \times U(1)_{L} \times U(1)_{R}$, and spontaneous breaking to $H=S U(2)_{V} \times U(1)_{V}$. We gauge the $S U(2)_{L} \times U(1)_{Y}$ standard model subgroup, and the $W$ and $Z$ then acquire mass from the usual Higgs boson. The leptons $(\nu, e)$ will cancel gauge anomalies of the quark sector.

The low energy physics of the quark sector is represented by an effective lagrangian describing the NGB's and gauge fields $A$. It will also contain the spin- 1 vector and axial-vector fields, denoted by $B$ which will be assumed to transform covariantly under $G^{\prime}$. The NGB's are contained in a chiral matrix field $U$.

Under a general infinitesimal transformation, $\epsilon$, of $G$ we have:

$$
\begin{aligned}
& \delta U=i \epsilon_{L}(\epsilon) U-i U \epsilon_{R}(\epsilon), \\
& \delta A=d \epsilon+i[\epsilon, A], \\
& \delta B=i[\epsilon, B] .
\end{aligned}
$$

Eq.(25) allows for the possibility of a nonlinear realization, e.g., $\delta U=i \epsilon U-i U \epsilon^{\prime}(\epsilon, U)$, with $\epsilon^{\prime} \in H$ [22]. If we specialize to $G=U\left(N_{f}\right)_{L} \times U\left(N_{f}\right)_{R}$, with the associated gauge bosons $A_{L}$ and $A_{R}$, and background fields $B_{L}$ and $B_{R}$ we have:

$$
\delta U=i \epsilon_{L} U-i U \epsilon_{R},
$$

and:

$$
\begin{array}{lll}
\delta A_{L}=d \epsilon_{L}+i\left[\epsilon_{L}, A_{L}\right], & & \delta A_{R}=d \epsilon_{R}+i\left[\epsilon_{R}, A_{R}\right], \\
\delta B_{L}=i\left[\epsilon_{L}, B_{L}\right], & & \delta B_{R}=i\left[\epsilon_{R}, B_{R}\right] .
\end{array}
$$

The full effective action contains the kinetic terms of the NGB's and gauge fields, and any mass terms associated with explicit breaking (which may involve the Higgs sector). The effective action also includes the WZW term, $\Gamma_{W Z W}(U, A+B)$, which represents the anomaly structure of the quark sector. We also have the contribution, $\Gamma_{\ell}$, to the effective action from the lepton sector.

The key point is that the covariant classical background $B$ fields are present in the quark sector, but not in the lepton sector. Mixed terms containing $A$ and $B$ will thus arise in the gauge anomalies of the quark sector, that are not cancelled by the lepton sector.

\section{A. The counterterm}

In deriving the counterterm, we will not need the explicit form of the WZW term, but only the consistent anomaly that it generates. Consider first the case $B=0$. Then under a general gauge transformation in $G^{\prime}$ we have:

$$
\delta \Gamma_{W Z W}=-2 \mathcal{C} \int \operatorname{Tr}\left[\epsilon\left(d A d A-\frac{i}{2} d A^{3}\right)\right] .
$$

The quantity $\mathcal{C}$ is fixed by properties of the underlying fermion theory. For example, for quarks transforming in the fundamental representation of $S U\left(N_{c}\right)$,

$$
\mathcal{C}=-\frac{N_{c}}{48 \pi^{2}} .
$$

Eq.(28) is the "consistent" form of the anomaly, before any counterterms are added, and it is cancelled by the contribution from the lepton sector:

$$
\delta \Gamma_{\ell}=2 \mathcal{C} \int \operatorname{Tr}\left[\epsilon\left(d A d A-\frac{i}{2} d A^{3}\right)\right] .
$$

It is convenient to write, modulo a total divergence,

$$
\delta \Gamma_{W Z W}=2 \mathcal{C} \int \operatorname{Tr}\left[d \epsilon\left(A d A-\frac{i}{2} A^{3}\right)\right] .
$$

Now we introduce the $B$ fields by making the replacement $A \rightarrow A+B$ in the quark sector only. This changes the variation of the WZW term so that under the general gauge transformation of Eq.(25) we have:

$$
\begin{aligned}
\delta\left(\Gamma_{W Z W}+\Gamma_{\ell}\right) & =2 \mathcal{C} \int \operatorname{Tr}\{d \epsilon[B d A+d A B+B d B \\
& -\frac{i}{2}\left(B A^{2}+A B A+A^{2} B\right) \\
- & \left.\left.\frac{i}{2}\left(B^{2} A+B A B+A B^{2}\right)-\frac{i}{2} B^{3}\right]\right\} .
\end{aligned}
$$


Our problem is to find a counterterm that cancels this variation.

The explicit construction of the counterterm is straightforward, and we obtain the result:

$$
\begin{aligned}
\Gamma_{c}= & -2 \mathcal{C} \int \operatorname{Tr}\left[(A d A+d A A) B+\frac{1}{2} A(B d B+d B B)\right. \\
& \left.-\frac{3 i}{2} A^{3} B-\frac{3 i}{4} A B A B-\frac{i}{2} A B^{3}\right] .
\end{aligned}
$$

The fact that $\delta \Gamma_{c}=-\delta\left(\Gamma_{W Z W}+\Gamma_{\ell}\right)$ can be verified explicitly. Therefore, the full action,

$$
\Gamma=\Gamma_{W Z W}+\Gamma_{c}+\Gamma_{\ell},
$$

is now gauge anomaly free in the presence of the NGB's, gauge fields and spin-1 mesons.

Note that if we specialize to $G=U\left(N_{f}\right)_{L} \times U\left(N_{f}\right)_{R}$, with the transformation law (27), the counterterm takes the form:

$$
\begin{aligned}
\Gamma_{c}= & -2 \mathcal{C} \int \operatorname{Tr}\left[\left(A_{L} d A_{L}+d A_{L} A_{L}\right) B_{L}\right. \\
& +\frac{1}{2} A_{L}\left(B_{L} d B_{L}+d B_{L} B_{L}\right)-\frac{3 i}{2} A_{L}^{3} B_{L} \\
& \left.-\frac{3 i}{4} A_{L} B_{L} A_{L} B_{L}-\frac{i}{2} A_{L} B_{L}^{3}\right]-(L \leftrightarrow R) .
\end{aligned}
$$

\section{B. Relation to Bardeen counterterm}

Suppose that we gauge only vector symmetries, for example $U(1)_{E M}$ in the standard model. We then have:

$$
\begin{aligned}
& A_{L} \rightarrow A+B_{L} \equiv \mathcal{A}_{L}, \\
& A_{R} \rightarrow A+B_{R} \equiv \mathcal{A}_{R},
\end{aligned}
$$

where $B_{L, R}$ are again background fields, transforming covariantly under the gauged symmetry. The Bardeen counterterm takes the form $-\Gamma_{W Z W}\left(U=1, \mathcal{A}_{L}, \mathcal{A}_{R}\right)$ from Eq.(69) (e.g., see Ref. [7]):

$$
\begin{gathered}
\Gamma_{\text {Bardeen }}=-\mathcal{C} \int \operatorname{Tr}\left[\left(d \mathcal{A}_{R} \mathcal{A}_{R}+\mathcal{A}_{R} d \mathcal{A}_{R}\right) \mathcal{A}_{L}\right. \\
-\left(d \mathcal{A}_{L} \mathcal{A}_{L}+\mathcal{A}_{L} d \mathcal{A}_{L}\right) \mathcal{A}_{R} \\
\left.-i\left(\mathcal{A}_{R}^{3} \mathcal{A}_{L}-\mathcal{A}_{L}^{3} \mathcal{A}_{R}+\frac{1}{2} \mathcal{A}_{R} \mathcal{A}_{L} \mathcal{A}_{R} \mathcal{A}_{L}\right)\right] .
\end{gathered}
$$

It can be easily verified that after including the counterterm (37), the full result $\Gamma_{W Z W}+\Gamma_{\text {Bardeen }}$ is gaugeinvariant in the vector subgroup. How does $\Gamma_{\text {Bardeen }}$ compare to our result in Eq. 355?

The Bardeen counterterm mixes the $B_{L}$ and $B_{R}$ fields. At first sight, this seems to contradict Eq.(35). However, upon closer inspection we see that all such mixed terms arrange themselves into operators that are gaugeinvariant in $A$. For example, the terms mixing $B_{L}$ and
$B_{R}$ with one $A$ field and two $B$ fields are:

$$
\begin{aligned}
& \operatorname{Tr}\left[\left(d B_{R} A+d A B_{R}+B_{R} d A+A d B_{R}\right) B_{L}\right] \\
- & \operatorname{Tr}\left[\left(d B_{L} A+d A B_{L}+B_{L} d A+A d B_{L}\right) B_{R}\right] \\
= & 3 \operatorname{Tr}\left[d A\left(B_{R} B_{L}-B_{L} B_{R}\right)\right],
\end{aligned}
$$

where a total divergence has been dropped. Terms with two $A$ 's and two $B$ 's are

$$
-3 i \operatorname{Tr}\left[A^{2}\left(B_{R} B_{L}-B_{L} B_{R}\right)\right] .
$$

Eqs.(38) and (39) combine into the gauge-invariant expression,

$$
3 \operatorname{Tr}\left[\left(d A-i A^{2}\right)\left(B_{R} B_{L}-B_{L} B_{R}\right)\right] .
$$

Continuing in a similar manner, the terms mixing $L$ and $R$ are all seen to form gauge-invariant operators in the fundamental gauge fields $A$.

Splitting the Bardeen counterterm into a gauge invariant and anomalous piece,

$$
\Gamma_{\text {Bardeen }}=\Gamma_{\text {Bardeen }}^{\text {G.I. }}+\Gamma_{\text {Bardeen }}^{\text {anom. }},
$$

we find:

$$
\begin{gathered}
\Gamma_{\text {Bardeen }}^{\text {G.I. }}=-\mathcal{C} \int \operatorname{Tr}\left\{3\left(d A-i A^{2}\right)\left(B_{R} B_{L}-B_{L} B_{R}\right)\right. \\
\left.+\left(D B_{R} B_{R}+B_{R} D B_{R}\right) B_{L}-\left(D B_{L} B_{L}+B_{L} D B_{L}\right) B_{R}\right\} .
\end{gathered}
$$

Here the covariant derivatives acting on $B_{L, R}$, taking account of the anticommuting forms, are

$$
\begin{aligned}
& D B_{L}=d B_{L}-i A B_{L}-i B_{L} A, \\
& D B_{R}=d B_{R}-i A B_{R}-i B_{R} A .
\end{aligned}
$$

The remaining, anomalous part can be simplified to:

$$
\begin{aligned}
& \Gamma_{\text {Bardeen }}^{\text {anom. }}=\Gamma_{c}=-\mathcal{C} \int \operatorname{Tr}[ \\
& 2(d A A+A d A) B_{L}+A\left(d B_{L} B_{L}+B_{L} d B_{L}\right) \\
- & \left.i\left(3 A^{3} B_{L}+\frac{3}{2} A B_{L} A B_{L}+A B_{L}^{3}\right)\right]-(L \leftrightarrow R) .
\end{aligned}
$$

When $A_{L}=A_{R}=A$, i.e., when only vector symmetries are gauged, our general expression Eq.(35) reduces to precisely this form. Our new counterterm is the generalization of the Bardeen counterterm when the gauge subgroup $G^{\prime}$ is not contained in the unbroken subgroup $H$ of the chiral theory $G / H$.

The Bardeen counterterm has been well-studied in the past. For example, in Ref. [23], the Bardeen counterterm is employed for the purpose of constructing the gauged WZW term by an integration formula that requires vanishing anomaly in the unbroken $(H)$ subgroup of a general $G / H$. Inclusion of the Bardeen counterterm can be 
phrased as the boundary condition $\Gamma(U=1)=0$ when integrating the anomaly [1]. Our results show, however, that this boundary condition is incompatible with gauge invariance in the general case involving non-vectorlike gauging.

The Bardeen counterterm has also appeared in phenomenological analyses [7]. However, such analyses neglect the important effects of neutral and charged weak currents, and have added the counterterm in an ad hoc manner: only photon gauge invariance is preserved, and global chiral symmetries are broken even in the absence of gauge fields. The Bardeen counterterm maintains gauge invariance in the presence of background fields only when vector symmetries are gauged. It is not the appropriate construct when the full standard model $S U(2)_{L} \times U(1)_{Y}$ gauging is relevant.

\section{GLOBAL CURRENT ANOMALIES AND GAUGE INVARIANT OPERATORS}

The new counterterm is necessary for a proper derivation of global current anomalies, such as the baryon current anomaly in the standard model. Our counterterm ensures that the action is anomaly free under the gauged $G^{\prime}$ symmetry, in the presence of arbitrary background fields. This action still has a number of global symmetries that are not broken explicitly by gauging, namely the special transformations for which $[\epsilon, A]=0$. The associated symmetry currents are generated by varying the background fields, and are conserved modulo anomalies. Since our theory is locally gauge invariant under $G^{\prime}$ transformations, the global anomalies generated from the full action will automatically be gauge covariant expressions in the $A$. In this sense, they are "covariant" anomalies. The formalism also implies that these currents and anomalies necessarily contain the background spin-1 meson fields, $B$. Note that since the global anomalies are derived from a well-defined action, they necessarily satisfy the appropriate extension of Wess-Zumino consistency conditions that describes variations with respect to both $A$ and $B$ fields.

\section{A. The general case}

Let us then consider the variation,

$$
\begin{aligned}
\delta U & =i \epsilon_{L}(\epsilon) U-i U \epsilon_{R}(\epsilon) \\
\delta A & =i[\epsilon, A]=0 \\
\delta B & =d \epsilon+i[\epsilon, B]
\end{aligned}
$$

Since $B$ enters only the "quark" sector, and we impose $[\epsilon, A]=0$, the lepton effective action, $\Gamma_{\ell}$, is invariant. We therefore need consider only the variation of $\Gamma_{W Z W}(U, A+B)+\Gamma_{c}(A, B)$.

We thus obtain the general expression for the global anomaly:

$$
\begin{gathered}
\delta\left(\Gamma_{W Z W}+\Gamma_{c}\right)=-2 \mathcal{C} \int \operatorname{Tr}\{\epsilon[ \\
3\left(d A-i A^{2}\right)^{2}+3\left(d A-i A^{2}\right) D B \\
\left.\left.+(D B)^{2}-\frac{i}{2} D\left(B^{3}\right)+i B\left(d A-i A^{2}\right) B-i\left(d A-i A^{2}\right) B^{2}\right]\right\},
\end{gathered}
$$

where $D B=d B-i A B-i B A$. Note the appearance of the covariant field strength, $\left(d A-i A^{2}\right)$. We emphasize that the form of this result depends on the condition $[\epsilon, A]=0$. In the explicit chiral representation for $U\left(N_{f}\right)_{L} \times U\left(N_{f}\right)_{R}$ the anomaly takes the form:

$$
\begin{aligned}
& \delta\left(\Gamma_{W Z W}+\Gamma_{c}\right)= \\
& -2 \mathcal{C} \int \operatorname{Tr}\left\{\epsilon _ { L } \left[3\left(d A_{L}-i A_{L}^{2}\right)^{2}+3\left(d A_{L}-i A_{L}^{2}\right)\left(D B_{L}\right)\right.\right. \\
& \quad+D B_{L} D B_{L}-\frac{i}{2} D\left(B_{L}^{3}\right)+i B_{L}\left(d A_{L}-i A_{L}^{2}\right) B_{L} \\
& \left.\left.\quad-i\left(d A_{L}-i A_{L}^{2}\right) B_{L}^{2}\right]\right\}-(L \leftrightarrow R),
\end{aligned}
$$

with $D B_{L}=d B_{L}-i A_{L} B_{L}-i B_{L} A_{L}$.

\section{B. Application to the Standard Model}

Let us illustrate the computation of covariant anomalies by considering the baryon current of the first generation quarks in the standard model. We will first give a description at the quark level, emphasizing that the counterterm is required for a correct derivation of the anomaly. We then give an equivalent description at the chiral lagrangian level. This leads to a generalization of the Goldstone-Wilczek current in the presence of gauge fields.

Let $Q=(u, d)$ and consider the action:

$$
\begin{aligned}
S_{Q}=\int d^{4} x \bar{Q}_{L}(i \not \partial & \left.+A_{L}+B_{L}\right) Q_{L} \\
& +\bar{Q}_{R}\left(i \not \partial+\not A_{R}+\not B_{R}\right) Q_{R}
\end{aligned}
$$

where $A_{L \mu}=g_{2} W_{\mu}^{a} \tau^{a} / 2+g_{1} W_{\mu}^{0} Y_{L} / 2, A_{R \mu}=$ $g_{1} W_{\mu}^{0} Y_{R} / 2, B_{L}=B_{R}=\omega_{\mu} \operatorname{diag}(1 / 3,1 / 3)$. Under variation in $\omega_{\mu}$ we obtain the baryon current:

$$
\frac{\delta S_{Q}}{\delta \omega_{\mu}}=J^{\mu}=\frac{1}{3} \bar{Q} \gamma^{\mu} Q
$$

By considering the local variation $\delta \omega=d \epsilon$, we obtain from the Weyl quark loops the consistent anomaly of the quark effective action:

$$
\begin{aligned}
& \left.\delta S_{Q}\right|_{\omega=0}= \\
& -2 \mathcal{C} \int \epsilon \operatorname{Tr}\left\{\frac{1}{3}\left[\left(d A_{L}\right)^{2}-\frac{i}{2} d\left(A_{L}^{3}\right)\right]\right\}-(L \leftrightarrow R) .
\end{aligned}
$$


We must also include the counterterm Eq.(35), which takes the form, to leading order in $\omega$ :

$$
\begin{aligned}
& \Gamma_{c}= \\
& 2 \mathcal{C} \int \operatorname{Tr}\left\{\frac{1}{3} \omega\left[A_{L} d A_{L}+d A_{L} A_{L}-\frac{3 i}{2} A_{L}^{3}\right]\right\}-(L \leftrightarrow R) .
\end{aligned}
$$

Under the local variation $\delta \omega=d \epsilon$ we find:

$$
\begin{aligned}
& \delta \Gamma_{c}= \\
& -2 \mathcal{C} \int \operatorname{Tr}\left\{\frac{1}{3} \epsilon\left[2 d A_{L} d A_{L}-\frac{3 i}{2} d\left(A_{L}^{3}\right)\right]\right\}-(L \leftrightarrow R),
\end{aligned}
$$

and upon combining the quark loop contribution with the counterterm we find:

$$
\begin{aligned}
& \delta S_{Q}+\left.\delta \Gamma_{c}\right|_{\omega=0}= \\
& -2 \mathcal{C} \int \operatorname{Tr}\left[\epsilon\left(d A_{L}-i A_{L}^{2}\right)^{2}\right]-(L \leftrightarrow R) \\
& =\int d^{4} x \epsilon \frac{1}{64 \pi^{2}} \epsilon_{\mu \nu \rho \sigma}\left(g_{2}^{2} F_{\mu \nu}^{a} F_{\rho \sigma}^{a}+\frac{1}{2} \operatorname{Tr} Y^{2} g_{1}^{2} F_{\mu \nu}^{Y} F_{\rho \sigma}^{Y}\right),
\end{aligned}
$$

where $F_{\mu \nu}^{a}=\partial_{\mu} W_{\nu}^{a}-\partial_{\nu} W_{\mu}^{a}+g_{2} \epsilon^{a b c} W_{\mu}^{b} W_{\nu}^{c}$ is the covariant $S U(2)_{L}$ field strength, and $F_{\mu \nu}^{Y}=\partial_{\mu} W_{\nu}^{0}-\partial_{\nu} W_{\mu}^{0}$ is the weak hypercharge field strength. The factor $\operatorname{Tr}\left(Y^{2}\right)=2 \times(1 / 3)^{2}-(4 / 3)^{2}-(-2 / 3)^{2}=-2$ is traced over the $(u, d)$ quarks. Note that we could also have read this result directly from Eq. (47).

Hence, with $\tilde{F}_{\mu \nu}=(1 / 2) \epsilon_{\mu \nu \rho \sigma} F^{\rho \sigma}$ :

$$
\partial_{\mu} J^{\mu}=-\frac{1}{32 \pi^{2}}\left(g_{2}^{2} F_{\mu \nu}^{a} \tilde{F}^{a \mu \nu}-g_{1}^{2} F_{\mu \nu}^{Y} \tilde{F}^{Y \mu \nu}\right) .
$$

While this result can be obtained by naively rescaling Feynman diagrams, using Adler's axial vector anomaly from QED as a starting point, the result would then be only fortuitously correct; the modified counterterm structure is required to generate the formally correct baryon current anomaly in the standard model. For $B \pm L$ we need a similar counterterm construction in the lepton sector with an auxiliary background field, etc.. Of course, the $B-L$ anomaly cancels between leptons and quarks, insofar as we take the limit of zero background fields after calculating the current divergence. The $B+L$ anomaly is 2 times the above result, Eq.(54).

Note that if we had inadvertently used the Bardeen counterterm of Eq.(37) in defining the baryon number current and divergence, we would have:

$$
\begin{aligned}
\Gamma_{\text {Bardeen }}=-2 \mathcal{C} \int & \operatorname{Tr}\left[\frac { 1 } { 3 } \omega \left(A_{L} d A_{L}-\frac{i}{2} A_{L}^{3}+3 d A_{R} A_{L}\right.\right. \\
- & \left.\left.A_{R} d A_{R}+\frac{i}{2} A_{R}^{3}-3 d A_{L} A_{R}\right)\right],
\end{aligned}
$$

and a short calculation shows that the resulting baryon number current would have zero divergence in place of $1 / 32 \pi^{2}$ in Eq.(54).

\section{Generalization of the Goldstone-Wilczek Current}

We can give an equivalent description of the anomalies at the level of the chiral theory of mesons, instead of at the quark level. The WZW term, with the new counterterm, can be expanded in the external fields $B$ :

$$
\Gamma(A, B, U)=\Gamma(A, U)+\int d^{4} x \operatorname{Tr}\left(B_{\mu} J^{\mu}\right)+\mathcal{O}\left(B^{2}\right) .
$$

The leading term in the expansion, $\Gamma(A, U)$, is the original WZW term. It generates the consistent gauge anomaly, which is cancelled by the leptons. Therefore the subsequent terms, such as $\operatorname{Tr}\left(B_{\mu} J^{\mu}\right)$, must be gauge invariant. In particular, $J_{\mu}$ is the covariant global current associated with $B$.

Let us again focus on the baryon number current in the $U(2)_{L} \times U(2)_{R}$ chiral theory, so that $B_{L}=B_{R}=$ $\omega \operatorname{diag}(1 / 3,1 / 3)$. First note that, in the limit $A_{L}=$ $A_{R}=0$ we see, from the WZW term reproduced below in Eq.(69), that: $\left(\alpha=d U U^{\dagger}\right)$

$$
\begin{aligned}
J_{\mu} & =\frac{\delta}{\delta \omega_{\mu}} \Gamma_{W Z W}=\frac{2}{3} \mathcal{C} \epsilon_{\mu \nu \rho \sigma} \operatorname{Tr}\left(\alpha^{\nu} \alpha^{\rho} \alpha^{\sigma}\right) \\
& =\frac{N_{c}}{72 \pi^{2}} \epsilon^{\mu \nu \rho \sigma} \operatorname{Tr}\left(U \partial^{\nu} U^{\dagger} U \partial^{\rho} U^{\dagger} U \partial^{\sigma} U^{\dagger}\right) .
\end{aligned}
$$

This is the Goldstone-Wilczek topological current that describes baryon number in the chiral lagrangian [11]. The current arises automatically upon introducing the background $\omega$ field into the WZW term. With $N_{c}=3$ it yields a baryon number of 1 for a Skyrmion hedgehog configuration.

Armed with our new counterterm and using Eqs.(55)69) we can compute the form of the baryon current in the presence of the gauge fields. For simplicity we keep just the $S U(2)_{L}$ part. The result is a gauge invariant current, as it must be, and for $N_{c}=3$ :

$$
J_{\mu}=-\frac{1}{24 \pi^{2}} \epsilon^{\mu \nu \rho \sigma} \operatorname{Tr}\left(\tilde{\alpha}_{\nu} \tilde{\alpha}_{\rho} \tilde{\alpha}_{\sigma}+\frac{3 i}{2} F_{L \nu \rho} \tilde{\alpha}_{\sigma}\right) .
$$

Here:

$$
\begin{aligned}
\tilde{\alpha}_{\mu} & =\left(D_{\mu} U\right) U^{\dagger}, \\
D_{\mu} & =\partial_{\mu}-i A_{L \mu}, \\
F_{L \mu \nu} & =i\left[D_{\mu}, D_{\nu}\right] .
\end{aligned}
$$

This current reduces to the Goldstone-Wilczek result when $A_{L} \rightarrow 0$.

A similar current using electromagnetic gauging was constructed by Callan and Witten, where the new term is seen to play a crucial role in monopole catalysis of baryon number viewed at the Skyrmion level [24]. The nonabelian form was constructed using dimensional deconstruction, matching to a Yang-Mills topological current in $D=5$ in Ref. [25]. The present derivation by variation of an action is more general, and it is now straightforward to construct any of the chiral topological currents 
by variation of the WZW term plus counterterm. This nonabelian $S U(2)_{L}$ current can arise only when we use the WZW term with our improved counterterm $\Gamma_{c}$.

Note that we can compute explicitly, for $N_{c}=3$,

$$
\partial_{\mu} J^{\mu}=-\frac{1}{16 \pi^{2}} \operatorname{Tr}\left(F_{L \mu \nu} \tilde{F}_{L}^{\mu \nu}\right)
$$

where a useful identity (the Bianchi identity for a deconstructed $D=5$ theory [21]) is:

$$
\left[D_{\mu}, \tilde{\alpha}_{\nu}\right]-\left[D_{\nu}, \tilde{\alpha}_{\mu}\right]=\left[\tilde{\alpha}_{\mu}, \tilde{\alpha}_{\nu}\right]-i F_{L \mu \nu}
$$

Eq.(59) reproduces the result for $S U(2)_{L}$ obtained in Eq.(54). Note that for a self-dual instanton in which $F_{\mu \nu}=\tilde{F}_{\mu \nu}$ and we have the Euclidean action $\left(1 / 2 g_{2}^{2}\right) \int \operatorname{Tr} F F=8 \pi^{2} / g_{2}^{2}$, the baryon charge is changed by one unit, and $B+L$ changes by 2 units, confirming the usual intuition.

It is interesting to contemplate the full background field $(B)$ structure of Eq.(47). Note that the $B$-field containing terms are a total divergence, and can be absorbed by a redefinition of the baryon current. However, they probably do have a physical role to play at high baryon density. Note that there is no $F^{Y} d \omega$ term in the baryon current anomaly, owing to $\operatorname{Tr}\left(Y_{L}-Y_{R}\right)=0$. However, if we keep the $\rho$ meson then there are surviving mixed isospin and weak-isospin terms $\operatorname{Tr}\left(F_{W} d \rho\right)\left(F_{W}\right.$ is weak isospin). Whether there is more to this story, e.g., an enhancement of baryon number violation at large finite baryon density through this form of the mixed anomaly, or a description of certain superfluid phases of baryons, remains to be investigated.

\section{Gauge invariant operators}

Throughout this discussion, we have implicitly assumed that the counterterm (33) is unique. In fact, we can construct additional gauge invariant operators beginning at quadratic order in the $B$ fields. While in the limit $B \rightarrow 0$ the gauge field part of the global anomaly is uniquely determined, these terms can potentially lead to an ambiguity in the structure of the global anomaly at finite $B$. For simple group models, we can add a general counterterm of the form,

$$
\Gamma_{\mathrm{c}, \text { G.I. }}=\int c_{1} \operatorname{Tr}\left[B^{2}\left(d A-i A^{2}\right)\right]
$$

with $c_{1}$ a free parameter. In fact, it is easy to show that the general anomaly (47) is not affected by this term.

For product group models, the situation is slightly more complicated. A short calculation, after dropping total derivatives, yields the general expression for term containing at least one $A$ field:

$$
\begin{aligned}
\Gamma_{\text {c, G.I. }}= & \frac{N_{c}}{24 \pi^{2}} \int\left\{c_{1 L} \operatorname{Tr}\left[B_{L}^{2}\left(d A_{L}-i A_{L}^{2}\right)\right]\right. \\
& +c_{2 L} \operatorname{Tr}\left(B_{L}\right) \operatorname{Tr}\left[B_{R}\left(d A_{R}-i A_{R}^{2}\right)\right] \\
& \left.+c_{3 L} \operatorname{Tr}\left(B_{L}\right) \operatorname{Tr}\left[B_{R} D B_{R}\right]\right\}+(L \leftrightarrow R) .
\end{aligned}
$$

To recover a parity-symmetric theory when only vector symmetries are gauged, we should have $c_{L}=-c_{R}$. The effects of these operators on the anomalies at finite $B_{L, R}$ can be worked out in the general case. For example, when the field $\omega$ coupling to baryon number is the only background field present, then $\Gamma_{c \text {, G.I. }}=0$, since $\epsilon^{\mu \nu \rho \sigma} \omega_{\mu} \omega_{\nu} \cdots=0$

\section{PSEUDO-CHERN-SIMONS TERMS FOR THE STANDARD MODEL}

We can apply the results from the previous section to compute the explicit form of the pCS terms for the standard model. For simplicity, we focus on a single standard model generation, i.e., the $(u, d)$ quarks and the $\left(\nu_{e}, e\right)$ leptons. The low energy physics of the quark sector is represented by a $U(2)_{L} \times U(2)_{R}$ chiral lagrangian describing interactions of the three pions and the $\eta$, gauge fields, and vector mesons. The lepton sector is also present in the effective theory. We introduce the full $S U(2)_{L} \times U(1)_{Y}$ gauging and gauge anomalies cancel between the quark and lepton sector. We also include the spin-1 vector mesons, treated as classical background fields, corresponding to the $\rho^{0, \pm}, \omega^{0}$ and $a_{1}^{0, \pm}, f_{1}^{0}$ vector and axial-vector mesons. At $\lesssim \mathrm{GeV}$ energies, where the chiral lagrangian description is appropriate, the $W$ and $Z$ bosons may be subsequently integrated out of the theory, and their effects represented by the corresponding charged and neutral weak currents.

The complete effective lagrangian thus contains the kinetic terms of the NGB's, leptons, gauge and vector meson fields, and any mass terms associated with symmetry breaking (which may involve the Higgs sector for the $S U(2)_{L} \times U(1)_{Y}$ breaking). The effective action also includes the WZW term and the counterterm:

$$
\Gamma_{W Z W}^{\text {full }}=\Gamma_{W Z W}(U, A+B)+\Gamma_{c}(A, B),
$$

which represent the full anomaly structure of the quark sector.

For the fundamental gauge fields we write

$$
\begin{aligned}
& A_{L}=g_{2} W^{a} \tau^{a} / 2+g_{1} W^{0}\left(\begin{array}{ll}
\frac{1}{6} & \\
& \frac{1}{6}
\end{array}\right), \\
& A_{R}=g_{1} W^{0}\left(\begin{array}{ll}
\frac{2}{3} & \\
& -\frac{1}{3}
\end{array}\right) .
\end{aligned}
$$

(we use $W^{0}$ to denote the $U(1)_{Y}$ gauge field, so as not to confuse with our previous usage of $B$ as a generic classical 
background field). In terms of the charge and mass eigenstates after electroweak symmetry breaking we have:

$$
\begin{aligned}
W^{0} & =-s_{W} Z+c_{W} A \\
W^{1} & =\frac{1}{\sqrt{2}} W^{+}+\frac{1}{\sqrt{2}} W^{-} \\
W^{2} & =\frac{i}{\sqrt{2}} W^{+}-\frac{i}{\sqrt{2}} W^{-} \\
W^{3} & =c_{W} Z+s_{W} A,
\end{aligned}
$$

where $c_{W}=g_{2} / \sqrt{g_{1}^{2}+g_{2}^{2}}, s_{W}=g_{1} / \sqrt{g_{1}^{2}+g_{2}^{2}}$.

Let us now put the standard model in the classical background of vector and axial-vector mesons:

$$
\begin{aligned}
& B_{V} \equiv B_{L}+B_{R}=g\left(\begin{array}{cc}
\rho^{0} & \sqrt{2} \rho^{+} \\
\sqrt{2} \rho^{-} & -\rho^{0}
\end{array}\right)+g^{\prime}\left(\begin{array}{ll}
\omega & \\
& \omega
\end{array}\right), \\
& B_{A} \equiv B_{L}-B_{R}=g\left(\begin{array}{cc}
a^{0} & \sqrt{2} a^{+} \\
\sqrt{2} a^{-} & -a^{0}
\end{array}\right)+g^{\prime}\left(\begin{array}{ll}
f & \\
& f
\end{array}\right) .
\end{aligned}
$$

where $\rho$ and $a$ are isotriplets and $\omega$ and $f$ are isosinglets. Note the slightly unconventional definitions $V, A=\left(A_{L} \pm A_{R}\right)$ instead of $V, A=\left(A_{L} \pm A_{R}\right) / 2$; the resulting normalization of $g, g^{\prime}$ can be more readily compared to the literature. Note also that this normalization implies that

$$
g_{\omega}=\frac{3}{2} g^{\prime}
$$

where $g_{\omega}$ multiplies the field that is coupled to baryon number [13]. In the following, we suppress coupling constants for the vector fields. We can recover the complete result with couplings by taking $(A, W, Z) \rightarrow$ $\left(g_{2} A, g_{2} W, g_{2} Z\right),(\rho, a) \rightarrow(g \rho, g a),(\omega, f) \rightarrow\left(g^{\prime} \omega, g^{\prime} f\right)$

\section{A. Fundamental gauge fields and anomaly cancellation}

The WZW term for $U\left(N_{f}\right)_{L} \times U\left(N_{f}\right)_{R} \rightarrow U\left(N_{f}\right)_{V}$ is given in a convenient form by Kaymakcalan, Rajeev and Schechter [7] 31]. In terms of:

$$
\mathcal{A}_{L, R}=A_{L, R}+B_{L, R}, \quad \mathcal{C}=-\frac{N_{c}}{48 \pi^{2}},
$$

we have:

$$
\begin{aligned}
& \Gamma_{W} \text { ZW }\left(U, \mathcal{A}_{L}, \mathcal{A}_{R}\right)=\Gamma_{0}(U)+\mathcal{C} \int \operatorname{Tr}\left\{\left(\mathcal{A}_{L} \alpha^{3}+\mathcal{A}_{R} \beta^{3}\right)-\frac{i}{2}\left[\left(\mathcal{A}_{L} \alpha\right)^{2}-\left(\mathcal{A}_{R} \beta\right)^{2}\right]\right. \\
& \quad+i\left(\mathcal{A}_{L} U \mathcal{A}_{R} U^{\dagger} \alpha^{2}-\mathcal{A}_{R} U^{\dagger} \mathcal{A}_{L} U \beta^{2}\right)+i\left(d \mathcal{A}_{R} d U^{\dagger} \mathcal{A}_{L} U-d \mathcal{A}_{L} d U \mathcal{A}_{R} U^{\dagger}\right) \\
& \quad+i\left[\left(d \mathcal{A}_{L} \mathcal{A}_{L}+\mathcal{A}_{L} d \mathcal{A}_{L}\right) \alpha+\left(d \mathcal{A}_{R} \mathcal{A}_{R}+\mathcal{A}_{R} d \mathcal{A}_{R}\right) \beta\right]+\left(\mathcal{A}_{L}^{3} \alpha+\mathcal{A}_{R}^{3} \beta\right) \\
& \quad-\left(d \mathcal{A}_{L} \mathcal{A}_{L}+\mathcal{A}_{L} d \mathcal{A}_{L}\right) U \mathcal{A}_{R} U^{\dagger}+\left(d \mathcal{A}_{R} \mathcal{A}_{R}+\mathcal{A}_{R} d \mathcal{A}_{R}\right) U^{\dagger} \mathcal{A}_{L} U \\
& \left.\quad+\left(\mathcal{A}_{L} U \mathcal{A}_{R} U^{\dagger} \mathcal{A}_{L} \alpha+\mathcal{A}_{R} U^{\dagger} \mathcal{A}_{L} U \mathcal{A}_{R} \beta\right)+i\left[\mathcal{A}_{L}^{3} U \mathcal{A}_{R} U^{\dagger}-\mathcal{A}_{R}^{3} U^{\dagger} \mathcal{A}_{L} U-\frac{1}{2}\left(U \mathcal{A}_{R} U^{\dagger} \mathcal{A}_{L}\right)^{2}\right]\right\} .
\end{aligned}
$$

Here $\alpha=d U U^{\dagger}$ and $\beta=U^{\dagger} d U$. The function $\Gamma_{0}$ is given by

$$
\Gamma_{0}(U)=-\frac{i \mathcal{C}}{5} \int_{M^{5}} \operatorname{Tr}\left(\alpha^{5}\right)=\frac{i N_{c}}{240 \pi^{2}} \int d^{5} x \epsilon^{A B C D E} \operatorname{Tr}\left(\alpha_{A} \alpha_{B} \alpha_{C} \alpha_{D} \alpha_{E}\right)
$$

where $M^{5}$ is a five dimensional manifold with spacetime as its boundary. The quantization condition ensures that $e^{i \Gamma_{0}}$ is independent of the choice of bounding surface. In four dimensions,

$$
\Gamma_{0}(U)=-\frac{2 N_{c}}{15 \pi^{2} f_{\pi}^{5}} \int_{M^{4}} \operatorname{Tr}\left[\pi(d \pi)^{4}\right]+\ldots .
$$

Also, we have the new counterterm $\Gamma_{c}$, which is given by Eq.(35). We now take $\Gamma_{W Z W}^{\text {full }}=\Gamma_{W Z W}+\Gamma_{c}$ and examine the terms containing mixed factors of fundamental gauge fields, $A$ 's, and classical background fields, $B$ 's. Notationally, in the following, e.g., a term denoted $\Gamma_{A A B B}$ contains two factors of $A$ and two factors of $B$, etc..

The terms in $\Gamma_{W Z W}^{\mathrm{full}}$ involving just the fundamental gauge fields $A_{L, R}$ do not involve the counterterm. Explicitly, we see that the terms with three and four fundamental fields read: 


$$
\Gamma_{A A A}=\mathcal{C} \int d Z A Z\left(\frac{2}{3} s_{W}-\frac{1}{2} \frac{s_{W}}{c_{W}^{2}}\right)+d A A Z\left(\frac{2}{3} \frac{s_{W}^{2}}{c_{W}}\right)+\left(d W^{+} W^{-}+d W^{-} W^{+}\right)\left(\frac{1}{6} \frac{s_{W}^{2}}{c_{W}} Z-\frac{1}{6} s_{W} A\right)
$$

$$
\Gamma_{A A A A}=\mathcal{C} \int i W^{+} W^{-} Z A\left(\frac{1}{4} \frac{s_{W}}{c_{W}}\right)
$$

sector loop contributions [20, 26].

The terms $\Gamma_{A A A}$ and $\Gamma_{A A A A}$ combine with the leptonsector loop contributions to produce gauge invariant operators that do not involve the vector meson fields. In a formal limit where we assume the leptons are heavy, we can integrate out $(\nu, e)$ to obtain the lepton-sector WZW term as a function of $W, Z, \gamma$ and the NGB's of the Higgs boson. In this case, the pure gauge terms Eqs.7273) cancel exactly against corresponding lepton

\section{B. Interactions involving vector meson fields}

Since the $B$ fields transform linearly under the gauge transformations, the sum of the remaining terms must be separately gauge invariant. For the various remaining terms in $\Gamma_{W Z W}^{\text {full }}$ the result is:

$$
\begin{aligned}
& \Gamma_{A A B}=\mathcal{C} \int d Z Z\left[\frac{s_{W}^{2}}{c_{W}^{2}} \rho^{0}+\left(\frac{3}{2 c_{W}^{2}}-3\right) \omega-\frac{1}{2 c_{W}^{2}} f\right]+d A Z\left[-\frac{s_{W}}{c_{W}} \rho^{0}-\frac{3 s_{W}}{c_{W}} \omega\right]+d Z\left[W^{-} \rho^{+}+W^{+} \rho^{-}\right] \frac{s_{W}^{2}}{c_{W}} \\
& -s_{W} d A\left[W^{-} \rho^{+}+W^{+} \rho^{-}\right]+\left(D W^{+} W^{-}+D W^{-} W^{+}\right)\left[-\frac{3}{2} \omega-\frac{1}{2} f\right], \\
& \Gamma_{A B B}=\mathcal{C} \int Z\left\{d \rho^{0}\left[-\frac{3}{2 c_{W}} \omega-\frac{s_{W}^{2}}{c_{W}} a^{0}+\left(-\frac{3}{2 c_{W}}+3 c_{W}\right) f\right]+d \omega\left[-\frac{3}{2 c_{W}} \rho^{0}+\left(-\frac{3}{2 c_{W}}+3 c_{W}\right) a^{0}-\frac{s_{W}^{2}}{c_{W}} f\right]\right. \\
& \left.+d a^{0}\left[\frac{s_{W}^{2}}{c_{W}} \rho^{0}+\left(\frac{3}{2 c_{W}}-3 c_{W}\right) \omega-\frac{1}{2 c_{W}} f\right]+d f\left[\left(\frac{3}{2 c_{W}}-3 c_{W}\right) \rho^{0}+\frac{s_{W}^{2}}{c_{W}} \omega-\frac{1}{2 c_{W}} a^{0}\right]\right\} \\
& +s_{W} d A\left(\rho^{0} a^{0}+3 \rho^{0} f+3 \omega a^{0}+\omega f+\rho^{+} a^{-}+\rho^{-} a^{+}\right)-\frac{s_{W}^{2}}{c_{W}} d Z\left(\rho^{+} a^{-}+\rho^{-} a^{+}\right) \\
& \left.+\frac{3}{2}\left[W^{+} D \rho^{-}+W^{-} D \rho^{+}\right)\right](-\omega+f)+\frac{3}{2}\left[W^{+}\left(-\rho^{-}+a^{-}\right)+W^{-}\left(-\rho^{+}+a^{+}\right)\right] d \omega \\
& +\frac{1}{2}\left[W^{+} D a^{-}+W^{-} D a^{+}\right](-3 \omega-f)+\frac{1}{2}\left[W^{+}\left(-3 \rho^{-}-a^{-}\right)+W^{-}\left(-3 \rho^{+}-a^{+}\right)\right] d f, \\
& \Gamma_{B B B}=\mathcal{C} \int 2\left[\left(\rho^{-} f+\omega a^{-}\right) D \rho^{+}+\left(\omega a^{+}+\rho^{+} f\right) D \rho^{-}+\left(\omega a^{0}+\rho^{0} f\right) d \rho^{0}+\left(\rho^{+} a^{-}+\rho^{-} a^{+}+\omega f+\rho^{0} a^{0}\right) d \omega\right], \\
& \Gamma_{A A A B}=\mathcal{C} \int i W^{+} W^{-} Z\left[3 c_{W} \omega+\left(c_{W}+\frac{1}{2 c_{W}}\right) f\right], \\
& \Gamma_{A A B B}=\mathcal{C} \int i\left\{W^{+} W^{-}\left[\frac{3}{2}\left(\rho^{0}+a^{0}\right) \omega-\frac{1}{2}\left(\rho^{0}-a^{0}\right) f\right]\right. \\
& +W^{+} Z\left[\left(\frac{3 c_{W}}{2}-\frac{1}{c_{W}}\right) \rho^{-} f-\frac{3 c_{W}}{2} \rho^{-} \omega-\frac{c_{W}}{2} a^{-} f+\frac{3 c_{W}}{2} \omega a^{-}\right] \\
& \left.+W^{-} Z\left[\left(-\frac{3 c_{W}}{2}+\frac{1}{c_{W}}\right) \rho^{+} f+\frac{3 c_{W}}{2} \rho^{+} \omega+\frac{c_{W}}{2} a^{+} f-\frac{3 c_{W}}{2} \omega a^{+}\right]\right\}, \\
& \Gamma_{A B B B}=\mathcal{C} \int i\left\{W^{+}\left[\rho^{-} \rho^{0}(\omega-2 f)-\rho^{-} \omega a^{0}+\rho^{0} \omega a^{-}+\omega a^{-} a^{0}\right]+W^{-}\left[\rho^{+} \rho^{0}(-\omega+2 f)+\rho^{+} \omega a^{0}-\rho^{0} \omega a^{+}-\omega a^{+} a^{0}\right]\right. \\
& \left.+Z\left[\frac{1}{c_{W}} \rho^{+} \rho^{-} \omega+\left(-2 c_{W}+\frac{1}{c_{W}}\right)\left(2 \rho^{+} \rho^{-} f+\rho^{+} \omega a^{-}-\rho^{-} \omega a^{+}\right)+\frac{1}{c_{W}} \omega a^{+} a^{-}\right]\right\} .
\end{aligned}
$$

These results use the abbreviated notation of differential forms, so that for example $\int d^{4} x \epsilon_{\mu \nu \rho \sigma} A^{\mu} B^{\nu} \partial^{\rho} C^{\sigma}=$ $\int A B d C$. Here we have defined covariant derivatives of 
the charged fields as

$$
\begin{aligned}
D W^{ \pm} & =d W^{ \pm} \mp i s_{W} A W^{ \pm}, \\
D \rho^{ \pm} & =d \rho^{ \pm} \mp i s_{W} A \rho^{ \pm}, \\
D a^{ \pm} & =d a^{ \pm} \mp i s_{W} A a^{ \pm} .
\end{aligned}
$$

Note that the photon always appear as a field strength, or as a covariant derivative acting on charged vector bosons or mesons, as required by gauge invariance. Gauge invariance in $W$ and $Z$ is not explicit, since we have not included terms involving the pion fields.

Some notable interactions in the above include the term from $\Gamma_{A A B}$ :

$$
-\frac{3 s_{W}}{c_{W}} g_{2}^{2} g^{\prime} \mathcal{C} \int \omega Z d A .
$$

This interaction was studied in Ref. [14] and mediates neutrino-photon interactions in nuclear matter. It should be noted that without including $\omega$ in the WZW term, the $\omega Z d A$ interaction can still be obtained from the assumption that the physical $\omega$ couples to the baryon current, $J_{\mu}$, through a phenomenological interaction of the form $g_{\omega} \omega^{\mu} J_{\mu}$. At low energies we must then use for $J_{\mu}$ the modified form of the Goldstone-Wilczek current in the presence of $Z$ and $A$ as dictated by gauge invariance and the new counterterm. This contains the $\omega Z d A$ interaction precisely as in Eq. (766).

Note that in $\Gamma_{A B B}$ we find a term:

$$
3 s_{W} g_{2} g g^{\prime} \mathcal{C} \int d A \rho^{0} f
$$

This term mediates the decay $f_{1} \rightarrow \rho^{0} \gamma$ and is in reasonable accord with experiment. We will study such interactions in more detail elsewhere 13 .

In $\Gamma_{B B B}$ we find

$$
2 g^{2} g^{\prime} \mathcal{C} \int\left(\omega a^{0} d \rho^{0}+\rho^{0} a^{0} d \omega\right) .
$$

Operators of this form have been studied in Ref. [27]. However, this term can be modified by the gauge invariant operators of Eq. (62) whose coefficients are not fixed solely by anomaly matching arguments.

\section{CONCLUSIONS}

In this paper we constructed the gauged WZW term for the standard model $S U(2)_{L} \times U(1)_{Y}$ gauge subgroup of the $U(2)_{L} \times U(2)_{R}$ chiral symmetry of the strong interactions. An essential ingredient in this construction was the demand that we maintain gauge invariance in the presence of background fields coupled to the $U(2)_{L} \times U(2)_{R}$ currents.

These background fields play two distinct but equally important roles. First, they allow us to define the currents of global symmetries through variation of the background fields. When applied to the (anomalous) baryon current this allowed us to derive a generalization of the Goldstone-Wilczek current in the presence of both fundamental gauge fields and background vector fields. Second, in environments where physical background fields are non-zero, such as at finite baryon density, we are led to a rich set of new interactions. For example, we find interactions that mediate neutrino-photon interactions in nuclear matter as discussed previously in Ref.[14].

A third role for these fields naturally suggests itself, namely to promote them to the dynamical vector meson fields of QCD in the spirit of vector meson dominance. We then have an interesting set of pCS terms which couple the vector mesons of QCD to fundamental gauge fields. A detailed analysis of the phenomenology of these terms will be presented elsewhere [13]. We mention here that the couplings we find lead to results for both the rate and polarization structure of the decay $f_{1} \rightarrow \rho^{0} \gamma$ which are in agreement with experiment. We emphasize that the processes resulting from the identification of the background fields with vector mesons are not to be thought of as interactions in the fundamental, underlying theory of quarks and leptons. Rather, they emerge in a low-energy effective description of QCD coupled to electroweak gauge fields.

Since in the real world the $W$ and $Z$ bosons are much heavier than the scale of QCD, the $W$ and $Z$ should be integrated out and replaced by the charged and neutral currents to which they couple. In the formal limit $g_{1,2} \rightarrow 0$, the $W$ and $Z$ are explicit degrees of freedom in the lowenergy theory, and the constraints of $S U(2)_{L} \times U(1)_{Y}$ gauge symmetry are explicit. Since we remain in a perturbative regime, the constraints survive at the physical values of these couplings. Restricting the weak currents to their components involving light fields then gives a low-energy effective Lagrangian which is valid for energies and momentum transfers up to the scale at which chiral perturbation theory breaks down, $4 \pi f_{\pi} \sim 1 \mathrm{GeV}$.

The centerpiece of our analysis is the derivation of a new counterterm which must be added to the WZW term in order to maintain gauge invariance in the presence of background fields. Once the counterterm is fixed, the full action provides a complete description of the global current anomalies of the theory, and also leads to a rich set of new interactions with many potentially important physical applications.

\section{Acknowledgments}

We thank Jon Rosner and Koichi Yamawaki for helpful discussions. Research supported by the U.S. Department of Energy grant DE-AC02-76CHO3000 and by NSF Grants PHY-00506630 and 0529954. 
[1] J. Wess and B. Zumino, "Consequences of anomalous Ward identities," Phys. Lett. B 37, 95 (1971).

[2] E. Witten, "Global Aspects Of Current Algebra," Nucl. Phys. B 223, 422 (1983).

[3] J. Steinberger, "On the use of subtraction fields and the lifetimes of some types of meson decay," Phys. Rev. 76, 1180 (1949).

[4] J. S. Bell and R. Jackiw, "A PCAC puzzle: $\pi^{0} \rightarrow \gamma \gamma$ in the sigma model," Nuovo Cim. A 60, 47 (1969).

[5] S. L. Adler, "Axial vector vertex in spinor electrodynamics," Phys. Rev. 177, 2426 (1969).

[6] W. A. Bardeen, "Anomalous Ward Identities In Spinor Field Theories," Phys. Rev. 184, 1848 (1969).

[7] O. Kaymakcalan, S. Rajeev and J. Schechter, "Nonabelian Anomaly And Vector Meson Decays," Phys. Rev. D 30, 594 (1984).

[8] A. Manohar and G. W. Moore, "Anomalous Inequivalence Of Phenomenological Theories," Nucl. Phys. B 243, 55 (1984).

[9] H. Kawai and S. H. H. Tye, "Chiral Anomalies, Effective Lagrangian And Differential Geometry," Phys. Lett. B 140, 403 (1984).

[10] C. T. Hill and R. J. Hill, "T-parity violation by anomalies," arXiv:0705.0697 [hep-ph], Phys. Rev. D, in press; C. T. Hill and R. J. Hill, "Topological physics of little higgs bosons," Phys. Rev. D 75, 115009 (2007).

[11] J. Goldstone and F. Wilczek, "Fractional Quantum Numbers On Solitons," Phys. Rev. Lett. 47, 986 (1981).

[12] See the review of M. Harada and K. Yamawaki, "Hidden local symmetry at loop: A new perspective of composite gauge boson and chiral phase transition," Phys. Rept. 381, 1 (2003), and references therein.

[13] J. A. Harvey, C. T. Hill and R. J. Hill, work in progress.

[14] J. A. Harvey, C. T. Hill and R. J. Hill, "Anomaly mediated neutrino-photon interactions at finite baryon density," arXiv:0708.1281 [hep-ph], Phys. Rev. Lett., in press.

[15] A. A. Aguilar-Arevalo et al. [MiniBooNE Collaboration], Phys. Rev. Lett. 98, 231801 (2007).

[16] N. Kaiser and U. G. Meissner, "Generalized hidden symmetry for low-energy hadron physics," Nucl. Phys. A 519, 671 (1990).

[17] F. Klingl, N. Kaiser and W. Weise, "Effective Lagrangian approach to vector mesons, their structure and decays," Z. Phys. A 356, 193 (1996).

[18] E. Truhlik, J. Smejkal and F. C. Khanna, "Electromagnetic isoscalar $\rho \pi \gamma$ exchange current and the anomalous action," Nucl. Phys. A 689, 741 (2001).

[19] E. D'Hoker and E. Farhi, "Decoupling A Fermion Whose Mass Is Generated By A Yukawa Coupling: The General Case," Nucl. Phys. B 248, 59 (1984).

[20] E. D'Hoker and E. Farhi, "Decoupling A Fermion In The Standard Electroweak Theory," Nucl. Phys. B 248, 77 (1984).

[21] C. T. Hill and C. K. Zachos, "Dimensional deconstruction and Wess-Zumino-Witten terms," Phys. Rev. D 71, 046002 (2005); C. T. Hill, "Exact equivalence of the D =
4 gauged Wess-Zumino-Witten term and the D = 5 YangMills Chern-Simons term," Phys. Rev. D 73, 126009 (2006); C. T. Hill, "Anomalies, Chern-Simons terms and chiral delocalization in extra dimensions," Phys. Rev. D 73, 085001 (2006).

[22] S. R. Coleman, J. Wess and B. Zumino, "Structure of phenomenological Lagrangians. 1," Phys. Rev. 177, 2239 (1969); C. G. Callan, S. R. Coleman, J. Wess and B. Zumino, "Structure of phenomenological Lagrangians. 2," Phys. Rev. 177, 2247 (1969).

[23] C. S. Chu, P. M. Ho and B. Zumino, "Non-Abelian Anomalies and Effective Actions for a Homogeneous Space G/H," Nucl. Phys. B 475, 484 (1996).

[24] C. G. Callan and E. Witten, "Monopole Catalysis Of Skyrmion Decay," Nucl. Phys. B 239, 161 (1984).

[25] C. T. Hill, "Topological solitons from deconstructed extra dimensions," Phys. Rev. Lett. 88, 041601 (2002).

[26] R. J. Hill, "SU(3)/SU(2): the simplest WZW term," in preparation.

[27] S. K. Domokos and J. A. Harvey, "Baryon numberinduced Chern-Simons couplings of vector and axialvector mesons in holographic QCD," Phys. Rev. Lett. 99, 141602 (2007).

[28] Weyl spinor triangle loops yield a finite and unambiguous form of the anomaly that satisfies the Wess-Zumino consistency condition, hence known as the consistent anomaly. Dirac fermions have ambiguities arising from the left-right mixing via the mass term. Bardeen obtains the consistent anomaly for massive Dirac fermions by imposing the left-right symmetry of the classical Dirac action on the result [6]. The counterterm can be viewed as a definition of the fermion loop. Throughout this paper we will always assume that the fermion loops explicitly generate the consistent anomaly, and that the counterterm is appended to the action, since this is the appropriate implementation for the Wess-Zumino-Witten term. The counterterm in the action can be seen as arising from a Chern-Simons term in a $D=5$ theory with chiral delocalization of fermions on boundary branes, compactifying the theory to $D=4[21]$.

[29] In the case of QED, the covariant anomaly is distinguished from the consistent anomaly only by the factor of 3 in its coefficient. In a Yang-Mills theory the consistent anomaly is not gauge covariant, while the covariant anomaly is [6].

[30] Interactions of this form are often referred to as "ChernSimons" terms, though technically this is a misnomer. Chern-Simons terms occur only in odd dimensions and generate anomalies on boundaries. In even $D$ we refer to terms like $V^{(1)} V^{(2)} d V^{(3)}$ as pseudo-Chern-Simons terms (or pCS terms) which generate anomalies in the bulk.

[31] We have the opposite overall sign to [7] because, given $D=d-i A$ and interpreting $\Gamma_{W Z W}$ as an action arising from the quark level action $\int \bar{q} i \not D q=\int A \cdot J+\ldots$ it must shift by $-\int \epsilon \partial \cdot J$ under a gauge transformation. Our choice of the sign of $\mathcal{C}$ ensures this. 